\title{
LA (NO) REGULACIÓN DE LA DESINFORMACIÓN EN LA UNIÓN EUROPEA. UNA PERSPECTIVA COMPARADA
}

RAÚL MAGALLÓN ROSA 
SUMARIO

1. INTRODUCCIÓN. LA EUROPA DE LAS DISTINTAS VELOCIDADES FRENTE A LAS FAKE NEWS. 2. DESINFORMACIÓN Y FAKE NEWS. ESTADO DE LA CUESTIÓN. 3. EUROPA ANTE LA DESINFORMACIÓN. CADA ELECCIÓN ES UN NUEVO DESAFÍO. 3.1. EL COMITÉ DE EXPERTOS DE LA UE. ENTRE EL PROCESO DELIBERATIVO Y LA TOMA DE DECISIONES. 3.2. LA POSTURA DE LA COMISIÓN EUROPEA. PRIMERO FAKE NEWS, DESPUÉS DESINFORMACIÓN. 4. LA REGULACIÓN DE LA DESINFORMACIÓN EN LOS DISTINTOS PAÍSES DE LA UE. 4.1. ALEMANIA. LA RESPONSABILIDAD JURÍDICA DE LAS EMPRESAS TECNOLÓGICAS. 4.2. FRANCIA. LA PROTECCIÓN DE LAS ELECCIONES FRENTE A LA DESINFORMACIÓN. 4.3. ESPAÑA Y LA NO REGULACIÓN. LOS LÍMITES SOCIALES DE LO LEGISLABLE. 4.4. REINO UNIDO. HACIA UNA REGULACIÓN INTEGRAL DE LA DESINFORMACIÓN. 4.4.1. LA REGULACIÓN DE LAS EMPRESAS TECNOLÓGICAS. 4.4.2. LEGISLAR Y PROTEGER LA CAMPAÑA ELECTORAL.5. CÓMO COMBATIR LA DESINFORMACIÓN. CONCLUSIONES Y PERSPECTIVAS DE FUTURO. 6. CONCLUSIONES. 


\title{
LA (NO) REGULACIÓN DE LA DESINFORMACIÓN EN LA UNIÓN EUROPEA. UNA PERSPECTIVA COMPARADA
}

\author{
RAÚL MAGALLÓN ROSA ${ }^{1}$ \\ Universidad Carlos III de Madrid
}

\section{INTRODUCCIÓN. LA EUROPA DE LAS DISTINTAS VELOCIDADES FRENTE A LA DESINFORMACIÓN}

El avance de la desinformación, la propaganda digital y las mediáticamente denominadas fake news se presentan como una serie de problemáticas que afectan a la calidad de nuestra esfera pública y que requieren de medidas legislativas, formativas y sociales que mitiguen sus efectos. El objetivo de este estudio es realizar un análisis comparativo en torno a las diferentes iniciativas tomadas por los países de la Unión Europea para combatir las nuevas formas de desinformación.

En los últimos años hemos visto cómo cada proceso electoral supone la actualización automática del software de la desinformación y la incorporación automática de nuevas formas, narrativas y formatos a las diferentes estrategias de propaganda digital.

Por otra parte, es importante destacar que bajo el paraguas semántico del concepto desinformación encontramos la difusión de contenidos falsos en redes sociales y plataformas, la publicidad segmentada, la utilización de plataformas para operaciones de influencia e injerencia extranjera, la amplificación de discursos del odio y propagandísticos a través de trolls y bots, así como contenidos de clickbait que buscan optimizar el consumo de las redes sociales.

Desde esta perspectiva se analizan en primer lugar las medidas tomadas por la Unión Europea ante el nuevo escenario y, posteriormente, se estudian las propuestas

\footnotetext{
${ }^{1}$ Raúl Magallón Rosa. Departamento de Periodismo y Comunicación Audiovisual de la Universidad Carlos III de Madrid. Facultad de Humanidades, Documentación y Comunicación. Universidad Carlos III de Madrid C/ Madrid, 126 Getafe (Madrid). Doctor Europeus por la Universidad Complutense de Madrid y el CNRS (Iresco, París). Email: raul.magallon@uc3m.es

ID ORCID: https://orcid.org/0000-0002-2236-7802.
} 
concretas llevadas a cabo por los diferentes países que han decidido apostar por una regulación en torno a esta nueva cultura de la desinformación ${ }^{2}$. En cualquier caso, y puesto que la expresión fake news está siendo utilizada en todo el mundo por actores políticos para atacar a periodistas e informaciones que van contra sus intereses, es importante destacar que este concepto está siendo desplazado progresivamente por el de desinformación.

Por otra parte, resulta pertinente recordar que el interés actual por esta problemática se vio acelerado por el avance las denominadas 'amenazas híbridas ${ }^{33}$ y el escenario ya apuntado por la UE en 2016 en el que señalaba que "la Unión, sus Estados miembros y sus ciudadanos están sometidos a una presión sistemática cada vez mayor para hacer frente a las campañas de información, desinformación e intoxicación y a la propaganda por parte de países y agentes no estatales, como organizaciones terroristas y delictivas transnacionales en los países vecinos" ${ }^{\text {. }}$

En la actualidad cuestiones como la alfabetización digital, medidas de seguridad nacional, el big data y la inteligencia artificial ${ }^{5}$, la limitación de la libertad de expresión, la regulación del discurso periodístico o la transparencia en la publicidad institucional convergen en el cajón de sastre de la legislación relacionada con la desinformación ${ }^{6}$.

\section{Como señala Rafa Rubio ${ }^{7}$ :}

"Las explicaciones del fenómeno son variadas: una herramienta de la movilización social; la creación artificial de un peligro que ofrezca una excusa para aumentar el control informativo del gobierno; el fin de la desintermediación; el precio de adoptar este tipo de plataformas sociales en las que el elemento económico es primordial o un aviso del fin del consenso sobre las instituciones básicas..."

Desde un punto de vista legislativo, hay que distinguir también entre aquellos países que afrontaron el nuevo escenario acordando el desarrollo de una nueva legis-

2 Magallón Rosa, R. (2019). Unfaking News. Cómo combatir la desinformación. Madrid. Pirámide.

3 Quintana, Y. (2016). Ciberguerra. Madrid. Catarata.

4 Sobre la comunicación estratégica de la Unión para contrarrestar la propaganda de terceros en su contra (2016/2030(INI)). Véase: http://www.europarl.europa.eu/doceo/document/A-8-2016-0290_ ES.html? redirect

5 Cotino, L. (2017). "Big data e inteligencia artificial. Una aproximación a su tratamiento jurídico desde los derechos fundamentales”. Dilemata, No. 24, 2017, págs. 131-150. Véase: https://www. dilemata.net/revista/index.php/dilemata/article/view/412000104/494

6 Robles, M. (2018). "La desinformación: una perspectiva internacional y europea”. Elecciones, gobierno abierto, información y 'fake news`. VI Congreso Internacional, Valencia 11 de marzo de 2019. Disponible en: https://www.youtube.com/watch?v=BfZGT_YRWq4

7 Rubio, R. (2019). "Los efectos de la posverdad en la democracia”. Revista de Derecho Político,

N. ${ }^{\circ} 103$, septiembre-diciembre 2018, págs. 191-228. Véase: http://revistas.uned.es/index.php/ derechopolitico/article/view/23201 
lación, aquellos que optaron por adaptar leyes ya existentes y los que, de manera cautelosa, crearon grupos de trabajo para redactar informes que ayudaran a entender la complejidad del fenómeno.

Para realizar el estudio se ha aplicado una metodología cualitativa, centrada en la observación y análisis del tipo de relaciones, debate y crítica en torno a las iniciativas presentadas por parte de los diferentes estados de la Unión Europea. El estudio se centra principalmente en cuatro países: Alemania, Francia, España y Reino Unido.

El trabajo pretende distinguir entre medidas legislativas, iniciativas de alfabetización digital, informes, grupos de trabajo, etc. así como entre medidas internas y externas. Es decir, aquellas destinadas a monitorizar las actividades de propaganda e injerencia de terceros países en los procesos electorales.

Para la realización de esta investigación se ha tomado como punto de partida el trabajo de Daniel Funke para la International Fact-Checking Network ${ }^{8}$, en el que ha realizado una labor de recopilación y mapeo de las distintas medidas tomadas en todo el planeta hasta la fecha.

En este, sentido, es importante subrayar que los países de la UE que han tomado alguna medida son: Bélgica, Croacia, Dinamarca, Francia, Alemania, Irlanda, Italia, España, Suecia y Reino Unido.

Países de la UE donde se han tomado medidas contra la desinformación.

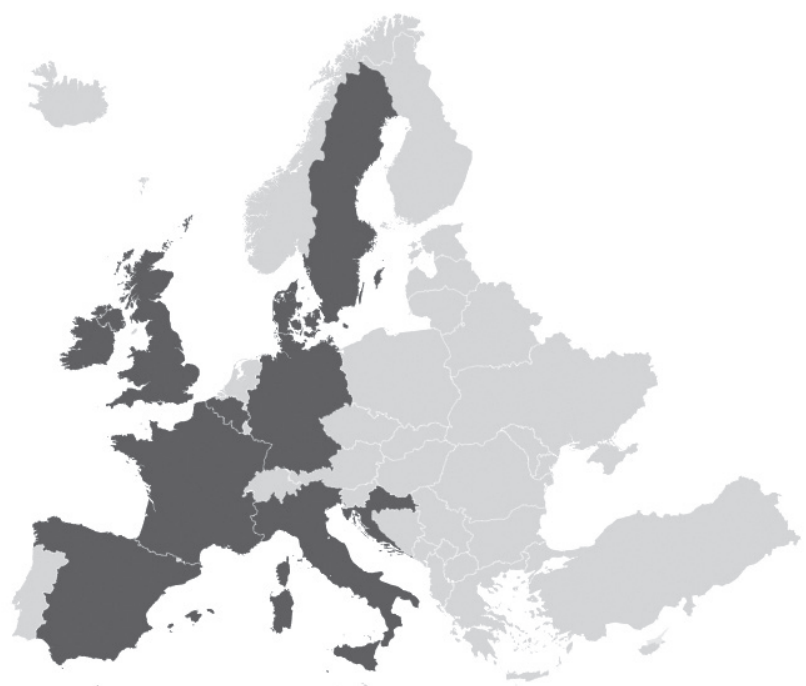

Fuente: Elaboración propia a partir de la base de datos creada por Daniel Funke (2019)

8 Funke, D. (2019). “A guide to anti-misinformation actions around the world”. Poynter. Febrero de 2019. Véase: https://www.poynter.org/fact-checking/2018/a-guide-to-anti-misinformationactions-around-the-world/ 
Ante las distintas elecciones que se celebran en la UE cada año, ésta decidió crear un grupo de expertos para el análisis de las fake news y la desinformación. En este contexto, Andrus Ansip — vicepresidente responsable del Mercado Único Digitalseñalaba:

"La desinformación, como instrumento de influencia política, no es ninguna novedad. Las nuevas tecnologías, especialmente las digitales, han ampliado su alcance a través del entorno en línea para socavar nuestra democracia y nuestra sociedad. Puesto que la confianza en línea es fácil de romper y difícil de recuperar, el sector necesita colaborar con nosotros en esta cuestión. Las plataformas en línea desempeñan un papel importante en la lucha contra las campañas de desinformación organizadas por personas y países que quieren poner en peligro nuestra democracia”.?

En este trabajo se profundiza en las medidas tomadas por Francia, Alemania e Inglaterra por representar tres modelos de sistemas mediáticos comparados ${ }^{10}$. Tomando como punto de partida este marco teórico, las preguntas de investigación son las siguientes:

- ¿Hay una coordinación entre los países de la Unión Europea a la hora de legislar las mediáticamente llamadas fake news?

- ¿Qué papel está llevando la Unión Europea en la lucha contra la desinformación?

- ¿Es necesario distinguir entre una legislación sobre los contenidos publicados y una regulación sobre la forma en la que se presentan esos contenidos?

- ¿Qué papel están llevando las empresas tecnológicas a la hora de implementar estas medidas legislativas?

- ¿Se puede hacer una tipología de medidas no legislativas tomadas por los distintos países?

Las hipótesis principales planteadas al inicio de este trabajo son las siguientes:

- Cada país afronta la regulación de la desinformación en función de su modelo de prensa y no tanto en relación a las recomendaciones de la UE. Estas nuevas regulaciones buscan un replanteamiento del papel del Estado como garante del pluralismo informativo.

- Una regulación restrictiva en cuanto a la libertad de información de las plataformas tecnológicas puede implicar un recorte posterior de libertades hacia los medios de comunicación.

9 Declaración de la Comisión Europea IP/18/3370 de 26 de abril de 2018. "Combatir la desinformación en línea: La Comisión propone un Código de Buenas Prácticas para toda la UE”. Véase: http://europa.eu/rapid/press-release_IP-18-3370_es.htm

${ }^{10}$ Hallin, D. y Mancini, P. (2004). Comparing Media Systems: Three Models of Media and Politics. Nueva York. Cambridge University Press. 
- La consideración de compañías tecnológicas — como son los casos de Google y Facebook - como empresas mediáticas modifica las formas de regulación mediáticas tradicionales.

En cualquiera de estos escenarios a analizar, la legislación digital presenta desafíos que se repiten: un modelo legislativo no adaptado a los cambios digitales, intereses de actores tradicionales que no quieren perder sus cuotas de mercado y poder, además del desconocimiento de fenómenos nuevos que rápidamente se instalan en nuestra cotidianeidad.

\section{DESINFORMACIÓN Y FAKE NEWS. ESTADO DE LA CUESTIÓN}

Para la Unión Europea, actor fundamental en su proceso de regulación social, "las noticias falsas consisten en una difusión intencional de la desinformación a través de plataformas sociales en línea, medios de difusión o impresión tradicional. El fenómeno tiene un impacto mayor que nunca, ya que es más fácil para cualquier persona publicar y compartir noticias o información en línea. Las redes sociales y las plataformas en línea desempeñan un papel importante para acelerar la difusión de estas noticias y permiten un alcance global sin mucho esfuerzo por parte del autor". ${ }^{11}$

En la actualidad, la desinformación parece querer producirse en una cantidad mucho mayor que el contenido generado por la verificación de hechos ${ }^{12} y$, junto a esta experiencia colectiva de desconfianza, ha emergido con fuerza y probablemente para quedarse siempre, el concepto y la cultura de la desinformación. Claire Wardle y Hossein Derakhshan publicaron en septiembre de 2017 un informe con el título El Desorden de la información: Hacia un marco interdisciplinario para la investigación y la formulación de políticas en el que señalaban:

"Nos abstenemos de utilizar el término 'fake news` por dos razones. En primer lugar, lamentablemente es inadecuado para describir el complejo fenómeno de la contaminación de la información. El término también ha comenzado a ser apropiado por los políticos de todo el mundo para describir a las organizaciones de noticias cuya cobertura encuentran desagradable. De esta manera, se está convirtiendo en el mecanismo por el cual los poderosos pueden atacar, restringir, socavar y burlar la prensa libre"13.

11 "Fake news and online disinformation". Véase: https://ec.europa.eu/digital-single-market/en/ fake-news-disinformation .

12 Shao, C. et alt. (2018). "The spread of low-credibility content by social bots". Nature Communications, 9: 4787. Disponible en: https://www.nature.com/articles/s41467-018-06930-7

13 Wardle, C. y Derakshan, H. (2017). "Information Disorder: Toward an interdisciplinary framework for research and policy making”. Council of Europe. Disponible en: https://rm.coe.int/ information-disorder-toward-an-interdisciplinary-framework-for-researc/168076277c 
En el informe, los autores distinguen entre misinformation (información errónea), disinformation (propaganda) y malinformation (desinformación):

- La misinformation o información errónea se produce cuando se comparte información falsa, pero no con la intención de hacer daño.

- La disinformation o propaganda se produce cuando se comparte información que es falsa y creada deliberadamente para dañar a una persona, grupo social, organización o país.

- La malinformation, entendida como una de las variantes de la desinformación, tiene lugar cuando se comparte información que se basa parcialmente en la realidad, utilizada para infligir daño a una persona, organización o país (discurso del odio o filtraciones).

Por su parte, Zuckerman incide en que "es un término vago y ambiguo que se extiende por todo: desde un equilibrio falso (noticias reales que no merecen nuestra atención), propaganda (discurso armado diseñado para apoyar a una parte sobre otra) y disinformatzya (información diseñada para sembrar duda y aumentar la desconfianza en las instituciones)" 14 .

En este sentido, y como aproximación metodológica, es importante señalar que existe el problema de la traducción del inglés de la palabra desinformación. La palabra desinformación en español hace referencia tanto a "falta de información, ignorancia" como a la acción y al efecto de desinformar — dar información intencionadamente manipulada al servicio de ciertos fines o bien dar información insuficiente u omitirla - En inglés, esta distinción — como hemos visto - se realiza a partir de tres términos: misinformation —información errónea-, disinformation, creada con una intencionalidad estratégica y malinformation, que contorsiona parcialmente la realidad y se asocia más a nuestra tradicional manipulación.

En los últimos años, las campañas electorales permanentes, la aparición de nuevos actores políticos y la pérdida de influencia de los medios de comunicación tradicionales en la consolidación de estructuras de debate han hecho que algunos países hayan optado por identificar la nueva legislación digital únicamente con nuestros derechos y deberes como ciudadanos con derecho a voto y no con la necesidad de establecer una esfera pública de calidad.

En este escenario, la regulación de la cultura de la desinformación se ha ido expandiendo por todo el globo. En 2018 fueron aprobadas o entraron en vigor leyes en países como Alemania, Canadá, Irlanda, Francia o Egipto ${ }^{15}$. Otros países, decidieron

${ }^{14}$ Zuckerman, E. (2017). "Stop Saying Fake News, It's not Helping” My Heart is in Accra. Disponible en: http://www.ethanzuckerman.com/blog/2017/01/30/stop-saying-fake-newsits-nothelping/

15 Haciyakupoglu, G. et alt. (2018). "Countering fake news. a survey of recent global initiatives”. RSIS. Disponible en: https:/www.rsis.edu.sg/wp-content/uploads/2018/03/PR180307_CounteringFake-News.pdf 
apostar por la alfabetización digital y la creación de grupos de acción ante posibles ataques exteriores.

En Australia, por ejemplo, el gobierno decidió crear un grupo de trabajo para identificar posibles ataques cibernéticos y campañas de influencia extranjera dirigidas a las siguientes elecciones australianas. En Asia, la ley más restrictiva ha venido de Malasia que penaliza la divulgación de "cualquier noticia, información, datos e informes, que sean total o parcialmente falsos" ${ }^{\text {"16. En }}$ Filipinas, como en el caso de Trump, el término fake news se utiliza para atacar a las voces críticas e incluso algunos estudios señalan que el presidente Duterte pudo usar bots automatizados y cuentas falsas de Facebook para amplificar su mensaje y llegar a la presidencia.

\section{EUROPA ANTE LA DESINFORMACIÓN. CADA ELECCIÓN ES UN NUEVO DESAFÍO}

Ante los resultados electorales, políticos y sociales que acontecimientos como el Brexit, el referéndum de paz en Colombia o las elecciones en Estados Unidos de 2016 habían (re)producido en la esfera pública, la UE decidió profundizar y estudiar el alcance y los distintos actores que formaban parte de esta nueva guerra informacional.

Identificar a los distintos actores del proceso —así como su parte de responsabilidad social percibida — era fundamental para poder dar posteriormente una respuesta colectiva.

Con esta intención, decidió lanzar una consulta pública para reunir las opiniones de una amplia gama de partes interesadas — que tuvo que ser respondida antes del 23 de febrero de 2018-. El proceso se completaba con encuentros de un grupo de expertos, eventos y un informe.

Del cuestionario realizado inicialmente para comprender el fenómeno es importante señalar que la UE hablaba en aquel momento de desinformación intencionada y distinguía las temáticas y problemáticas de la siguiente manera: políticas, salud, medio ambiente, inmigración, seguridad o economía.

También preguntaba por los mecanismos de diseminación con un mayor impacto a la hora de difundir las fake news y daba la opción de escoger entre: influencers/ líderes de opinión, bots, usuarios de redes sociales, algoritmos y decisiones editoriales. Es decir, incluía a los medios de comunicación en la consulta sobre las distintas formas de desinformación.

${ }^{16}$ Head, J. (2018). "Outlaw or ignore? How Asia is fighting 'fake news"”. BBC, 4 de abril de 2018. Disponible en: https://www.bbc.com/news/world-asia-43637744 


\subsection{El Comité de expertos de la UE. Entre el proceso deliberativo y la toma de decisiones}

En marzo de 2018, se hizo público el informe final del grupo de expertos de la UE sobre 'Fake News y Desinformación digital ${ }^{17}$. Según el resultado consensuado, la respuesta que había que dar se establecía sobre cinco pilares:

1. Mejorar la transparencia de las noticias online.

2. Promover la alfabetización mediática e informativa.

3. Desarrollar herramientas para empoderar a usuarios y periodistas.

4. Salvaguardar la diversidad y la sostenibilidad del ecosistema de los medios de comunicación europeos.

5. Promover la investigación continua sobre el impacto de la desinformación en Europa.

Por otra parte se hacía un llamamiento para que todas las partes interesadas — con un papel relevante-, incluidas las plataformas en línea, las organizaciones de medios de comunicación (prensa, cadenas de radio y televisión, etc.), periodistas, verificadores datos, creadores de contenido independiente y la industria de la publicidad se comprometieran con un Código de Buenas Prácticas.

El informe también hablaba de identificar y mapear las fuentes de desinformación y los mecanismos que contribuyen a su amplificación digital, además de proporcionar un espacio seguro para acceder y analizar plataformas y datos con el objetivo de tener una mejor comprensión del funcionamiento de los algoritmos. Sin olvidar, por supuesto, la necesidad de compartir conocimientos con medios de comunicación y plataformas para mejorar la conciencia pública sobre la desinformación.

Respecto al Código de Buenas Prácticas, el trabajo señalaba que las plataformas debían adaptar sus políticas publicitarias —incluyendo la adhesión al principio de 'seguir el dinero' - , al tiempo que se evitaban los incentivos que conducen a la desinformación. De este modo, según los expertos, se podría desalentar la diseminación y amplificación de la desinformación con fines de lucro.

En este sentido, las plataformas debían garantizar la transparencia y la responsabilidad en relación al procesamiento de los datos para espacios publicitarios, con fechas claras de vencimiento, respeto a la privacidad, libertad de expresión, etc.

Además, el estudio destacaba que las plataformas debían garantizar que el contenido patrocinado — incluida la publicidad política — se distingue adecuadamente de otro contenido. De este modo, las plataformas deberían tomar las medidas necesarias para habilitar el acceso a los datos — cumpliendo con las necesidades de privacidad - para actividades de verificación y fact-checking.

17 "Final report of the High Level Expert Group on Fake News and Online Disinformation". Disponible en: https://ec.europa.eu/digital-single-market/en/news/final-report-high-level-expert-groupfake-news-and-online-disinformation 
Las plataformas tenían que poner a disposición de sus usuarios avanzados configuraciones y controles para empoderarlos y personalizar su experiencia en línea, tomar medidas efectivas para mejorar la visibilidad de noticias confiables y facilitar a los usuarios acceso a ella.

Sin embargo, varios miembros del comité de expertos, incluida Clara Jiménez de Maldita.es, lanzaron un comunicado alternativo en el que destacaban que "si bien la desinformación es claramente un problema, su escala e impacto, los agentes asociados a la misma y las estructuras de amplificación no se han investigado ni examinado adecuadamente" 18 .

Al mismo tiempo, subrayaban que "en un momento en el que muchos actores políticos parecen creer que la solución a la desinformación en internet es una ley contra las fake news, el informe claramente especifica que no lo es, instando a la cautela y señalando su escepticismo hacia cualquier regulación de contenido”.

$\mathrm{Y}$ apuntaban a que nuestro conocimiento actual se centra "casi exclusivamente en los datos de EEUU”, por lo que era vital que la UE recopilara datos de estudios transfronterizos para comprender las diferencias y matices en el alcance, la escala y el impacto de la desinformación en los 28 estados miembros. De esta forma se planteaban como medidas preventivas la monitorización de la escala, de las técnicas, herramientas, naturaleza e impacto de la desinformación en la sociedad.

\subsection{La postura de la Comisión Europea. Primero fake news, después desinformación}

El informe de la Comisión Europea se dio a conocer el 26 de abril de 2018 tras analizar las conclusiones del Comité de Expertos. En él se defendía el desarrollo de un Código de Buenas Prácticas para las plataformas en cuestiones sobre: transparencia, contenidos patrocinados, claridad sobre el funcionamiento de los algoritmos, fuentes alternativas de información, etc.

Ese código debía estar consensuado inicialmente en julio de 2018 —aunque finalmente se anunció para septiembre de 2018 - y, en un primer momento, las plataformas en línea deberían desarrollar sus principios y seguirlo. En su respuesta pública, la UE suprimía el concepto fake news de su vocabulario y destacaba que se habían contabilizado hasta 18 procesos electorales donde la desinformación y la manipulación había estado presente dentro de la Unión Europea.

${ }^{18}$ Jiménez, C. et alt. (2018). "Seis puntos claves del informe sobre desinformación del grupo de expertos de la Comisión europea”. Medium, 12 de marzo de 2018. Disponible en: https://medium.com/@ hlegresponse/seis-puntos-claves-del-informe-sobre-desinformaci\% C3\% B3n-del-grupo-de-expertos-dela-comisi\%C3\%B3n-europea-6191483db548 
Con el objetivo de dar respuesta a estas inquietudes y tendencias, la Comisión proponía una serie de medidas para combatir la desinformación en internet. Entre esas medidas cabe citar las siguientes ${ }^{19}$ :

1. Un Código de Buenas Prácticas sobre desinformación.

2. Una red europea independiente de verificadores de información.

3. Una plataforma europea en línea segura sobre desinformación que sirviera de apoyo para la red de verificadores de información y para los investigadores universitarios.

4. Reforzar la alfabetización mediática.

5. Apoyar a los Estados miembros para asegurar la resiliencia de las elecciones.

6. Promoción de sistemas voluntarios de identificación electrónica para reforzar la rastreabilidad y la identificación de los proveedores de información.

7. Apoyar una información plural y de calidad.

8. Una política de comunicación estratégica coordinada.

El 26 de septiembre de 2018, la comisaria de Economía Digital y Sociedad Mariya Gabriel anunciaba la finalización del borrador de Código de Buenas Prácticas afirmando: "Esta es la primera vez que la industria acuerda un conjunto de normas de autorregulación para combatir la desinformación en todo el mundo, de forma voluntaria. La industria se está comprometiendo con una amplia gama de acciones, desde la transparencia en la publicidad política hasta el cierre de cuentas falsas y la desmonetización de proveedores de desinformación" ${ }^{20}$.

Los firmantes de este Código, finalmente se habían comprometido a tomar medidas en cinco áreas:

1. Interrumpir los ingresos por publicidad de ciertas cuentas y sitios web que difunden la desinformación.

2. Hacer que la publicidad política sea más transparente.

3. Abordar el problema de las cuentas falsas y los robots en línea.

4. Empoderar a los consumidores para que informen sobre la desinformación y accedan a diferentes fuentes de noticias — al tiempo que mejoran la visibilidad y la posibilidad de encontrar contenido autorizado-.

19 Declaración de la Comisión Europea IP/18/3370 de 26 de abril de 2018. "Combatir la desinformación en línea: La Comisión propone un Código de Buenas Prácticas para toda la UE". Véase: http://europa.eu/rapid/press-release_IP-18-3370_es.htm

${ }^{20}$ Declaración de la Comisión Europea IP/18/ 5914 de 26 de septiembre de 2018. "Statement by Commissioner Gabriel on the Code of Practice on Online Disinformation". Véase: http://europa.eu/ rapid/press-release_STATEMENT-18-5914_en.htm 
5. Empoderar a la comunidad de investigadores para monitorizar la desinformación en línea a través de un acceso compatible con la privacidad a los datos de las plataformas.

En diciembre de 2018 la UE, presentó un plan de acción para intensificar los esfuerzos para contrarrestar la desinformación tanto en Europa como fuera de la UE. Andrus Ansip, señalaba:

"Debemos estar juntos y aunar fuerzas para proteger nuestras democracias frente a la desinformación. Hemos observado tentativas de interferir en las elecciones y referéndums, y las pruebas apuntan a Rusia como principal fuente de esas campañas. Para hacer frente a estas amenazas, proponemos mejorar la coordinación con los Estados miembros a través de un sistema de alerta rápida, reforzar nuestros equipos dedicados a desenmascarar la desinformación, aumentar el apoyo a los medios de comunicación y a los investigadores e instar a las plataformas online a cumplir sus compromisos. Luchar contra la desinformación requiere un esfuerzo colectivo"21.

Para ello, se contemplaba dotar al Grupo Especial sobre Comunicación Estratégica y a la Célula de Fusión contra las Amenazas Híbridas del Servicio Europeo de Acción Exterior (SEAE) —así como a las delegaciones de la UE en los países vecinos- de efectivos especializados y de nuevas herramientas de análisis. Se preveía también que se duplicara el presupuesto para comunicación estratégica del SEAE destinado a combatir la desinformación y sensibilizar sobre sus efectos — pasando de 1,9 millones de euros en 2018 a 5 millones de euros en 2019 - y se instaba a los Estados miembros de la UE a complementar estas medidas reforzando sus propios medios de lucha contra la desinformación. Por otra parte, se anunciaba que el sistema de alerta rápida debía estar en funcionamiento para marzo de 2019 pensando en las elecciones europeas de mayo.

Ese mismo mes se conocía la puesta en marcha de FactCheckEU, un proyecto de colaboración entre los firmantes europeos del código de principios de la Red Internacional de Verificación de Información de cara a las elecciones europeas de mayo de 2019. El proyecto, que contó con 19 medios europeos de 13 países diferentes, se presentaba como un recurso pero también como una incógnita para mantener la estabilidad democrática dentro de la Unión Europea.

21 Declaración de la Comisión Europea IP/18/ 6647 de 5 de diciembre de 2018. "Una Europa que protege: la UE intensifica la lucha contra la desinformación”. Véase: http://europa. eu/rapid/press-release_IP-18-6647_es.htm 


\section{LA REGULACIÓN DE LA DESINFORMACIÓN EN LOS DISTINTOS PAÍSES DE LA UE}

Las relaciones Prensa-Estado se han caracterizado tradicionalmente por el desempeño de tres funciones por parte de los Estados: censor, regulador y protector, siendo estas dos últimas las que han prevalecido en los países de regímenes democráticos ${ }^{22}$.

La mayoría de las veces cuando se habla de regulación — sobre todo desde el sector periodístico — saltan las alarmas y la palabra se asocia a cualquier tiempo pasado en el que la intención del partido o político de turno era la de limitar la libertad de expresión e información. Sin embargo, legislar también puede significar adaptarse a nuevos escenarios, formatos y actores así como a los nuevos desafíos que se plantean para seguir defendiendo el pluralismo informativo.

Desde esta perspectiva, y como bien apunta Myriam Redondo al referirse a España, "tratamos de solucionar con nuevas leyes problemas que ya caen bajo el paraguas de normativa existente, pero que no se aplica o se aplica a discreción: el acoso en línea, la usurpación de identidad, los ataques a la dignidad o al honor, la xenofobia"23.

Aunque es indudable el acierto de esta afirmación, también lo es que el encaje legal de los nuevos usos sociales de la tecnología puede producir nuevos fenómenos. En este sentido, la regulación de la cultura de la desinformación se puede plantear desde, al menos, dos perspectivas ${ }^{24}$ :

1. La necesidad de regular los contenidos independientemente de la forma en la que éstos se presentan.

2. La necesidad de legislar la forma en la que se presentan los contenidos como un mecanismo de transparencia y de defensa de nuestros derechos como consumidores.

Estas dos perspectivas conviven en las iniciativas legislativas analizadas. Desde el punto de vista de la orientación de las medidas tomadas por los distintos países hasta ahora, Funke distingue entre: concienciación, sanción, monitorización y rendición de cuentas $^{25}$.

En este sentido, las medidas y acciones tomadas por los distintos países de la UE son las siguientes:

${ }^{22}$ Sanmartí, J.M. et alt. (2010a). “Análisis del modelo desregulador de prensa en España frente al modelo liberal británico y al intervencionista euromediterráneo”. Textual and Visual Media. Madrid. VOL. 3, 2010. http://textualvisualmedia.com/index.php/txtvmedia/article/view/125

23 Redondo, M. (2018). "Falacias en red. La ilusión del muro". Eldiario.es, 17 de marzo de 2018. Disponible en: https://www.eldiario.es/tribunaabierta/Falacias-red-ilusion-muro_6_751084896.html

24 Magallón-Rosa, R. (2018). “Leyes fake news”. Telos. Madrid: Fundación Telefónica. Disponible en: https://telos.fundaciontelefonica.com/las-leyes-las-fake-news-problema-la-libertad-informacion-nolegislar/

25 Funke, D. (2019). “A guide to anti-misinformation actions around the world”. Poynter. Febrero de 2019. Disponible en: https://www.poynter.org/fact-checking/2018/a-guide-to-anti-misinformationactions-around-the-world/ 


\begin{tabular}{|l|l|}
\hline \multicolumn{1}{|c|}{ País } & \multicolumn{1}{c|}{ Tipo de acción } \\
\hline Bélgica & Grupo de expertos \\
\hline Bélgica & Campaña de alfabetización mediática \\
\hline Croacia & Iniciativa legislativa \\
\hline Dinamarca & Campaña de alfabetización mediática \\
\hline Dinamarca & Grupo de trabajo gubernamental \\
\hline Dinamarca & Plan de acción gubernamental \\
\hline Francia & Ley \\
\hline Alemania & Ley \\
\hline Irlanda & Iniciativa legislativa \\
\hline Italia & Portal online para reportar sobre desinformación \\
\hline Italia & Informe de las autoridades \\
\hline España & Recomendaciones de un comité \\
\hline España & Grupo de trabajo sobre ciberseguridad \\
\hline Suecia & Autoridad gubernamental \\
\hline Reino Unido & Grupo de trabajo gubernamental \\
\hline Reino Unido & Informe parlamentario \\
\hline
\end{tabular}

Fuente: Elaboración propia a partir de la base de datos creada por Daniel Funke (2019)

Como se puede observar en nuestro entorno, y frente a las recomendaciones de la UE, cada país está afrontando la problemática legal incidiendo en diferentes soluciones — muy determinadas por intereses específicos—. En Irlanda, donde también se ha incluido una regulación en torno a los bots, primero decidieron definir los distintos actores del proceso.

Un 'bot' sería reconocido en la legislación irlandesa como cualquier elemento de software que utiliza 25 o más cuentas o perfiles en línea para ejecutar tareas automatizadas. En diciembre de 2017, Irlanda hizo público que el uso de 'bots' de internet para influir en el debate político sería castigado con multas de hasta $10.000 €$ o cinco años de prisión. ${ }^{26}$

Suecia, por su parte, anunció la creación de nueva autoridad pública encargada de contrarrestar la desinformación ante posibles operaciones de influencia.

En Italia, y antes de la celebración de las elecciones de 2018, el gobierno pidió a la ciudadanía que denunciara en un portal específico las posibles infor-

${ }^{26}$ Legislación redactada por el partido conservador Fianna Fáil. 
maciones falsas que la ciudadanía encontrara en internet y que pudieran afectar a ese proceso electoral ${ }^{27}$.

En Bélgica, por su parte, se apostó por la alfabetización mediática y el gobierno lanzó un sitio web para informar a la ciudadanía sobre información errónea e implementar un sistema de propuestas para combatirlo ${ }^{28}$.

En Dinamarca, dónde se creó un grupo de trabajo responsable de desarrollar respuestas a campañas generalizadas de desinformación y ataques desde el extranjero, se ha apostado por la alfabetización mediática y se ha establecido un plan de acción gubernamental para coordinar a los distintos actores implicados de cara a las siguientes elecciones.

Más recientemente, el gobierno croata anunció en enero de 2019 que está trabajando en una ley para detener la propagación del discurso de odio y la información errónea en las plataformas de redes sociales. El proyecto de ley, enfocado principalmente a Facebook, trata de reforzar la alfabetización digital en lugar de sancionar la propagación de información errónea o falsa.

Lo interesante del debate es que en esa normativa se podría considerar que el discurso de odio pudiera ser un área de regulación más natural para los legisladores en Europa que para la propia legislación croata.

\subsection{Alemania. La responsabilidad jurídica de las empresas tecnológicas}

Dentro de la UE, la legislación alemana es considerada un modelo de referencia. Alemania, optó por un modelo regulatorio orientado directamente a los canales tecnológicos de distribución de la desinformación.

Inicialmente, Facebook, Twitter y Google acordaron eliminar dicho contenido de sus plataformas en un plazo de 24 horas, en virtud de un acuerdo de 2015 con el gobierno alemán. Sin embargo, un informe de 2017 encargado por el Ministerio de Justicia encontró que las empresas seguían incumpliendo sus compromisos. A los efectos de la nueva ley, las 'plataformas en línea' serían definidas como cualquier sitio web —incluidas las redes sociales o los motores de búsqueda_ que contaran con más de 10.000 visitantes mensuales únicos.

En la actualidad, en Alemania las redes y los medios sociales deben suprimir los discursos de odio, fake news y material ilegal o se enfrentan a penas de 40 millones de euros. La Ley de Aplicación de la Red (Network Enforcement Act) ${ }^{29}$, comúnmente conocida como la 'Ley de Facebook', fue aprobada por el Bundestag —el organismo

27 "Protocollo Operativo per il contrasto alla diffusione delle Fake News attraverso il webin occasione della Campagna elettorale per le Elezioni politiche 2018”. Véase: https://www. commissariatodips.it/fileadmin/src/doc/pdf/comunicato18012018.pdf

28 “Comment arrêter les fake news?". Véase: https://monopinion.belgium.be/processes/ stopfakenews

29 "Act to Improve Enforcement of the Law in Social Networks". Véase: https://www.bmjv.de/ SharedDocs/Gesetzgebungsverfahren/Dokumente/NetzDG_engl.pdf?_blob=publicationFile\&v=2 
parlamentario de Alemania- en junio de 2017 y entró en vigor en octubre de 2017. Desde el 1 de enero de 2018 los grandes agentes de internet están sometidos a la NetzDG, la normativa que obliga a plataformas con más de dos millones de usuarios en el país a retirar contenidos que pudieran ser constitutivos de delitos en menos de 24 horas desde la recepción de la denuncia por parte de un usuario.

Al respecto, el ministro de justicia alemán Heiko Maas señalaba: "la experiencia ha demostrado que, sin presión política, los grandes operadores de plataformas no cumplirán sus obligaciones, y esta ley es por lo tanto imperativa”.

La ley se limita a las redes y medios sociales únicamente, es decir a aquellas plataformas donde el contenido de cualquier tipo se puede compartir libremente y hacerse público, y no a plataformas como WhatsApp que a priori permiten una comunicación interpersonal no pública.

A la hora de prever las sanciones, la legislación distingue también según el alcance de las mismas: plataformas con más de 20 millones de usuarios en Alemania (hasta la fecha solo Facebook) podrían ser multadas con cifras que oscilan entre 2,5 y 40 millones de euros, plataformas entre 4 y 20 millones de usuarios alemanes (por ejemplo, Youtube o Instagram) serían multadas con entre 1 y 25 millones euros; y por último, plataformas con 2-4 millones de usuarios alemanes - tales como Twitter- que podrían ser multadas con cifras que varían entre los 250.000 euros y los 15 millones de euros.

Desde entonces países como Rusia, Singapur o Filipinas han citado directamente la ley alemana como un ejemplo positivo al contemplar o proponer el desarrollo de una legislación para eliminar el contenido 'ilegal en línea.

\subsection{Francia. La protección de las elecciones frente a la desinformación}

En Francia, la proposición de ley presentada a la Asamblea Nacional el 21 de marzo de 2018 relativa a la lucha contra las falsas informaciones señalaba en su exposición de motivos que "los recientes acontecimientos electorales han demostrado la existencia de campañas masivas para difundir información falsa destinadas a modificar el curso normal del proceso electoral a través de los servicios de comunicación en línea".

El texto utilizaba la noción de fausse information frente a la tradicional en Francia de fausse nouvelle. Una distinción importante desde el punto de vista de los límites que establece y de la (no) profesionalización de la misma. La proposición se planteaba desde tres ejes:

1. Nuevas herramientas para luchar mejor contra la diseminación de informaciones falsas durante el período electoral.

2. Imponer mayores obligaciones de transparencia para plataformas como Facebook con el fin de permitir a las autoridades públicas detectar posibles campañas para desestabilizar a las instituciones mediante la difusión de in- 
formaciones falsas y, por otra parte, a los usuarios conocer al anunciante del contenido patrocinado.

3. Designar un juez específico en periodo electoral para detener la difusión de informaciones falsas y que debe tomar una decisión en un plazo de 48 horas.

El proyecto de ley facultaba a la autoridad —El Consejo Superior de lo Audiovisual_para suspender unilateralmente la licencia de cualquier “organización mediática de influencia extranjera” durante una campaña electoral nacional (una clara alusión a Rusia, pero que también podría aplicarse teóricamente a EEUU y Google o Facebook si éstas fueran consideradas en un futuro empresas mediáticas). También exigía que las plataformas y las redes sociales divulguen las identidades de los anunciantes que pagan por distribuir contenido, así como las cantidades que están pagando.

La proposición — tras pasar por la Comisión de Asuntos Culturales — reformuló los objetivos de la ley al señalar que "la noción de manipulación de la información corresponde tanto a las disposiciones como a las cuestiones relacionadas con el texto". De esta forma se alejaba de una posible regulación de las informaciones satíricas y humorísticas. Las enmiendas a la ley ${ }^{30}$ empezaban señalando que "más amplia que la noción de 'noticias falsas', la 'falsa información' se refiere a la información que carece de cualquier elemento factual controlable que pueda hacerla plausible”. Por lo tanto, según las enmiendas, no era intencional ni eficaz atraer opiniones o artículos satíricos en esta categoría jurídica.

Sin embargo, permanecía una de las propuestas más controvertidas: en los tres meses previos a las elecciones presidenciales era posible el cierre de medios extranjeros.

Finalmente la ley fue aprobada en noviembre de 2018 y permite a los jueces ordenar, durante las elecciones, la inmediata eliminación de los artículos en línea que decidan que constituye desinformación. En diciembre, la ley fue validada por el Consejo Constitucional ${ }^{31}$.

\subsection{España y la no regulación. Los límites sociales de lo legislable}

La regulación de la desinformación ha de tener en cuenta el ecosistema informativo en el que se integran. En diciembre de 2017, el Partido Popular anunciaba una proposición no de ley sobre fake news ${ }^{32}$. En la misma, se instaba al Gobierno —en aquel momento del Partido Popular - a impulsar la elaboración de métodos para

30 “Justice: lutte contre la manipulation de l'information". Véase: http://www.assemblee-nationale. fr/15/amendements/0799/CION-CEDU/AC16.asp

31 Décision n 2018-773 DC du 20 décembre 2018 "Loi relative à la lutte contre la manipulation de l'information”. Véase: https:/www.conseil-constitutionnel.fr/decision/2018/2018773DC.htm

32 "Proposición no de Ley relativa al impulso de las medidas necesarias para garantizar la vercidad de las informaciones que circulan por servicios conectados a internet y evitar injerencias que pongan en 
determinar la veracidad de informaciones en internet y se proponía identificarlas mediante un sellado que sirviera de aviso ante los ciudadanos de 'potenciales informaciones falsas. Además señalaba que al tratarse de amenazas que podían afectar "a la seguridad y bienestar sociales”, estos métodos y medidas de acción deberían ser desarrollados por instituciones públicas especializadas que actuaran en colaboración con distintos actores.

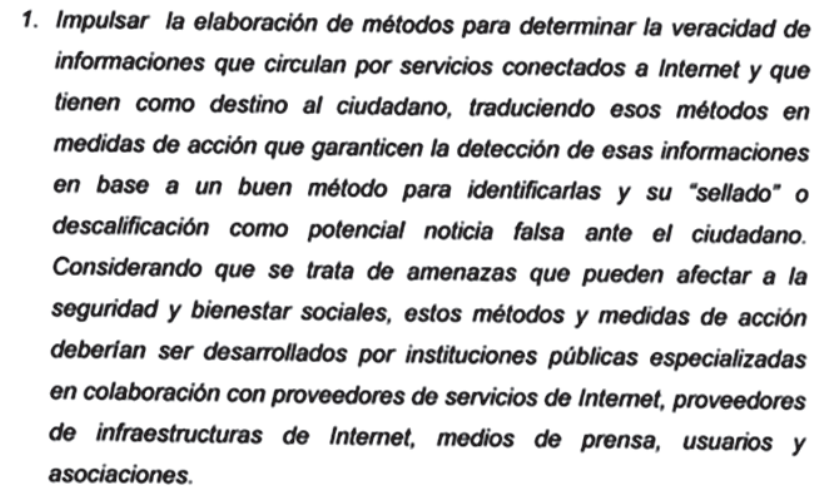

Fuente: Grupo Parlamentario Popular en el Congreso de los Diputados.

La propuesta también generaba dudas razonables puesto que abría la puerta a que los "servicios encargados de la seguridad pública" desarrollaran sistemas de vigilancia, monitorización, mitigación y respuesta ante 'acciones extranjeras'. Por otra parte, en un contexto creciente de ciberguerra ${ }^{33}$ y conflicto diplomático en red que describía la proposición no de ley se instaba a reforzar la comunicación estratégica en la UE frente a aquellas campañas de desinformación con objetivos de desestabilización — planificadas y gestionadas por actores con intenciones hostiles-.

En marzo de 2018 se confirmaba que el pleno del Congreso había rechazado la iniciativa del Partido Popular para luchar contras las fake news (noticias falsas). Poco después, y a través de la Comisión de Seguridad Nacional del Congreso de los Diputados, se aprobaba una proposición no de ley que instaba al Gobierno a reforzar los medios materiales y humanos dedicados a combatir las campañas de desinformación en el ciberespacio. Dicha proposición señalaba:

"La extensión de las actividades de desinformación al resto de la Unión hacen aconsejable que, sin descuidar las actuales tareas del East Stratcom Task Force, se am-

peligro la estabilidad institucional de España". Véase: http://www.gppopular.es/wp-content/ uploads/2017/12/171219-PNL-Noticias-falsas.pdf

33 Quintana, Y. (2016). Ciberguerra. Catarata. Madrid. 
plíe su rango de acción a las actividades de desinformación en el seno de la Unión Europea; algo que ya se hizo en algún momento a la vista de algunas desinformaciones fomentadoras de la islamofobia en Alemania".

E instaba al gobierno a:

1 Participar de manera activa en el proceso de construcción de la estrategia de la UE para detener la difusión de noticias falsas en línea.

2. Impulsar que, sin perjuicio de los objetivos actualmente asignados a la East Stratcom Task Force, se ampliara su rango de acción a las campañas de desinformación que pudieran ser detectadas respecto a los Estados miembros.

3. Desplegar las acciones necesarias para proceder al refuerzo inmediato en medios humanos y materiales del East Stratcom Task Force perteneciente al Servicio de Acción Exterior Europeo.

En marzo de 2019, se conocía que el entonces gobierno en funciones había activado una unidad contra la desinformación de cara a las elecciones del 28 de abril que integraba a expertos del Departamento de Seguridad Nacional, de la Secretaría de Estado de Comunicación y de aquellos ministerios más concernidos como son interior, defensa o asuntos exteriores. ${ }^{34}$

\subsection{Reino Unido. Hacia una regulación integral de la desinformación}

Reino Unido es quizá el país que — más allá de Estados Unidos— ha comprobado la magnitud y el alcance que tiene la desinformación en los procesos electorales. En Febrero de 2019, la Cámara de los Comunes hacia público su informe final sobre "Desinformación y fake news". ${ }^{35} \mathrm{El}$ informe estaba redactado por el Comité Digital de Cultura, Medios y Deporte —que había trabajado durante 18 meses para comprender la dimensión del problema- y abogaba por:

1. Un código ético obligatorio para empresas de tecnología (supervisadas por un regulador independiente).

2. Un regulador que tuviera poderes reales para iniciar acciones legales contra compañías que infrinjan el código.

3. La reforma, por parte del gobierno, de leyes y normas vigentes en materia de comunicaciones electorales en relación a la participación extranjera en las elecciones del Reino Unido.

34 Abellán, L. (2019). "El Gobierno activa una unidad contra la desinformación ante las elecciones". El País, 11 de marzo de 2019. Disponible en: https://elpais.com/politica/2019/03/10/ actualidad/1552243571_703630.html

35 Véase: https://www.parliament.uk/business/committees/committees-a-z/commons-select/ digital-culture-media-and-sport-committee/news/fake-news-report-published-17-19/ 
4. La obligación por parte de las compañías tecnológicas de eliminar las fuentes reconocidas por su contenido dañino, incluidas aquellas fuentes que se haya comprobado que están dedicadas a la desinformación.

Para su realización el Comité había invitado a sus sesiones a parlamentarios de Argentina, Bélgica, Brasil, Canadá, Francia, Irlanda, Letonia o Singapur y coincidía en cambiar — como ya ocurrió con la UE - el título del mismo y añadir la palabra "desinformación" al informe final. Un fenómeno que los parlamentarios definieron como "la creación deliberada y el intercambio de información falsa y / o manipulada con la intención de engañar y confundir a las audiencias, ya sea con el propósito de causar daño o con fines políticos, personales o económicos”.

El informe estaba dividido en las siguientes áreas: la definición, rol y responsabilidades legales de las plataformas; el mal uso de los datos de Facebook y Cambridge Analytica, las campañas electorales, la influencia rusa en campañas políticas y la alfabetización digital.

\subsubsection{La regulación de las empresas tecnológicas}

El papel de las empresas tecnológicas en el nuevo marco normativo es quizá una de las cuestiones más complejas de abordar. El informe del Comité Digital de Cultura, Medios y Deporte destacaba que Facebook había violado intencionadamente y a sabiendas las leyes de privacidad de datos y las leyes contra la competencia.

Al respecto, Damian Collins, presidente de la Comisión señalaba: "Las grandes empresas tecnológicas no están cumpliendo con el deber de cuidado que deben a sus usuarios para actuar contra el contenido dañino y respetar su derecho de privacidad con los datos”.

Lo más destacado de las conclusiones finales es que recomendaba una nueva categoría para Facebook y Twitter: compañía de medios sociales. El objetivo era reforzar las responsabilidades de las empresas de tecnología, sin vincularlas necesariamente a los conceptos jurídicos y sociales de "plataforma" o "editor".

Esta idea es importante porque uno de los problemas de la ley francesa es que las medidas aprobadas que afectaban a las empresas tecnológicas — si un día algún país o la UE decide cambiar su forma jurídica y empezar a considerarlas empresas mediáticas_- podrían tener un efecto legislativo que afecte a la libertad de información de los propios medios de comunicación.

Otra cuestión que se abordaba era el de las cámaras de eco y la segmentación de los algoritmos. En 2017, el Reino Unido anunció un nuevo Centro de Ética de Datos e innovación para asesorar sobre cómo habilitar y garantizar la ética, los usos seguros e innovadores de datos y la inteligencia artificial.

Desde esta perspectiva, cuestiones como el monopolio de datos, el uso de algoritmos predictivos en la vigilancia policial, el uso de análisis de datos en campañas 
políticas o la posibilidad de sesgo en las decisiones de contratación automatizadas eran presentadas como problemáticas a resolver por este centro.

\subsubsection{Legislar y proteger la campaña electoral}

Como hemos visto hasta ahora, la manera de abordar una nueva legislación de los procesos electorales ha de partir de los sistemas jurídicos y mediáticos específicos de cada país. Sin embargo, las problemáticas que se repiten de manera global están haciendo que su encaje jurídico pueda tener elementos de uniformidad. Desde esta perspectiva, Damian Collins señalaba:

"Tenemos que aceptar que nuestras regulaciones electorales están totalmente desfasadas para la era de Internet. Necesitamos una reforma para que los mismos principios de transparencia de las comunicaciones políticas se apliquen en línea, tal como lo hacen en el mundo real. Se debe hacer más para exigir a los donantes principales que establezcan claramente la fuente de sus fondos".

En este sentido, el informe destacaba que la Comisión Electoral debería tener más poderes, incluido el derecho legal de obligar a las empresas tecnológicas a proporcionar información bajo su requerimiento. Por otra parte, apuntaba a que ésta - como órgano con capacidad de acción- debería tener el poder de aumentar el tamaño de las multas en función del volumen de negocios de una empresa.

El Comité encontró también evidencias sólidas que apuntaban a actores estatales hostiles que influyen en los procesos democráticos y señalaba que "el Reino Unido es claramente vulnerable a las campañas de influencia digital encubiertas”. Desde esta perspectiva, indicaba que el Gobierno debería realizar un análisis para comprender el alcance - por parte de actores extranjeros - sobre los votantes durante las elecciones pasadas - en una clara alusión al Brexit pero también a las elecciones generales de 2017 o al referéndum en Escocia de 2014-.

El informe recomendaba que el gobierno británico debería analizar las formas en las que la ley del Reino Unido ha de definir las campañas digitales, incluyendo tener definiciones acordadas de lo que constituye publicidad política en línea — tales como los tipos de palabras diseñados estratégicamente que aparecen continuamente en anuncios que no están patrocinados por un partido político específico-

Como novedad, el trabajo de la Cámara señalaba que también debe haber un reconocimiento del papel y el poder de las campañas no pagadas y de los grupos de Facebook que influyen en las elecciones y en los referendos (tanto dentro como fuera del período designado), además de una revisión exhaustiva de las normas y regulaciones actuales que rodean el trabajo político en los procesos electorales.

Para ello abogaban por aumentar la duración del período regulado, definir lo que constituye una campaña política y reducir el tiempo para que las devoluciones por los gastos electorales se envíen a la Comisión Electoral. 
Por otra parte, y como hemos indicado, se señalaba que el Gobierno debería explorar formas que permitieran que se pudieran otorgar más poderes a la Comisión Electoral para llevar a cabo su trabajo de manera integral, incluidas las siguientes medidas:

1. El derecho legal a obligar a las organizaciones que actualmente no están reguladas —incluidas las compañías de medios sociales - a proporcionar información relevante para sus consultas.

2. Aumentar el límite máximo actual de la multa de la Comisión Electoral de $20.000 £$ y cambiarse a una multa basada en un porcentaje fijo de facturación, en línea con los poderes ya conferidos a otros reguladores estatutarios - como sucede con Alemania-.

3. Desarrollar la capacidad de la Comisión Electoral de presentar una petición contra una elección debido a acciones ilegales $-\mathrm{y}$ que actualmente solo puede ser presentada por un individuo-.

4. Establecer y definir la capacidad de la Comisión Electoral de intervenir o detener a alguien que actúe ilegalmente en una campaña si vive fuera del Reino Unido.

Como hemos descrito, lo más interesante del modelo propuesto es que ponía énfasis en el periodo electoral — como ocurre con la ley francesa-, otorga más poderes a la Junta Electoral y distingue entre plataformas tecnológicas y medios sociales, pero también define la relevancia de la desinformación por el alcance de los mensajes — las multas a las compañías tecnológicas se establecerían dependiendo del número de usuarios en el país_-

\section{CÓMO COMBATIR LA DESINFORMACIÓN. PERSPECTIVAS DE FUTURO}

Como hemos visto, dentro del paraguas semántico de la cultura de la desinformación encontramos la difusión de la desinformación en redes sociales y plataformas, la elaboración de microperfiles políticos, la explotación de redes sociales para operaciones de influencia extranjera, la amplificación de discursos del odio o contenidos ofensivos a través de cuentas falsas o robots políticos así como contenidos de clickbait que buscan optimizar el consumo de las redes sociales ${ }^{36}$. Por esta razón, medidas como la alfabetización digital deberían entrar dentro de cualquier iniciativa promovida por los países de la UE para combatir la desinformación.

${ }^{36}$ Howard, P. et alt. (2018). "The IRA, Social Media and Political Polarization in the United States, 2012-2018." Working Paper 2018. Oxford, UK: Project on Computational Propaganda. Disponible en: https://comprop.oii.ox.ac.uk/research/ira-political-polarization/ 
Desde esta perspectiva, uno de los problemas que, como apuntábamos, se plantean es la falta de cohesión de la UE en esta cuestión. Al respecto, resulta pertinente señalar que la dificultad en la acción se reproduce porque ni siquiera aquellas soluciones que son más tecnológicas que políticas o culturales se están haciendo de manera coordinada. Como se ha explicado anteriormente, cada país ha funcionado tradicionalmente con unas relaciones prensa-estado diferentes y, en la última década, también con una (no) intervención estatal específica en la regulación de las grandes corporaciones tecnológicas.

Sin embargo, más allá de las propuestas formales conjuntas, parece que cada estado las va incorporando a su análisis geopolítico a diferente velocidad. Anya Schiffrin, directora del área de Tecnología, Medios y Comunicación de la Universidad de Columbia, señalaba al respecto que "es muy difícil que la UE logre unanimidad en este ámbito, por eso es más probable que los países, por separado, elaboren sus propias leyes”37.

Independientemente de una (no) regulación, no se explica que países como España no hayan organizado grupos de trabajo de expertos externos en la materia para conocer el estado de cuestión y las distintas aristas de un problema en permanente mutación. Como se ha demostrado, hasta ahora, los actores implicados en los procesos de desinformación electoral están aprendiendo nuevas estrategias con cada elección. En este sentido - y como han señalado los distintos informes elaborados por la UEel problema de la desinformación es que es una amenaza evolutiva y escalable que requiere de esfuerzos constantes de coordinación entre los distintos actores políticos e institucionales.

Por otra parte, y como han confirmado los estudios más recientes, la tipología de rumores, propaganda y falsos mensajes que se integran en la cultura de la desinformación dependen en parte de cada país ${ }^{38}$. Desde este punto de vista, un primer debate aparece a la hora de establecer una dimensión temporal y de intensidad legislativa en relación al papel de la desinformación en el desarrollo de los procesos democráticos:

1. Una visión más restrictiva considera que se ha de desarrollar una legislación más específica para los procesos electorales que impida cualquier desestabilización (como ocurre con las encuestas electorales).

2. Una visión más holística —y casi filosófica-, entiende que el concepto de fake news hace referencia a fenómenos diversos y muchas veces contradictorios y que, precisamente, durante el periodo electoral la protección de la libertad de (des)información y expresión deben estar más garantizada si cabe.

37 Alandete, D. (2018). "La Comisión Europea renuncia a la legislación contra las noticias falsas". El País, 3 de abril de 2018. Disponible en: https://elpais.com/internacional/2018/04/02/ actualidad/1522694360_002636.html

${ }^{38}$ Humprecht, E. (2018). "Where 'fake news' flourishes: a comparison across four Western democracies". Information, Communication E Society. Disponible en: https://www.tandfonline.com/doi/ful 1/10.1080/1369118X.2018.1474241 
En cualquier caso, parece evidente que la adaptación jurídica al nuevo escenario de desinformación está poniendo sobre la mesa la necesidad de una vertebración y coordinación de los diferentes organismos de los estados que han de velar porque las votaciones se celebren de manera segura — aun sabiendo que las injerencias y ataques exteriores son más que probables—.

Por otra parte, resulta importante definir los distintos actores y reguladores que intervienen en un proceso que se va transformando elección tras elección. El informe del Parlamento Británico señalaba que Facebook, en particular, no está dispuesto a ser responsable ante los reguladores de todo el mundo y que el gobierno británico debería considerar el impacto de tales monopolios en el mundo político y en la democracia. En este sentido, Collins, presidente de la comisión británica encargada de redactar el informe final sobre "Desinformación y fake news" afirmará:

"Necesitamos un cambio radical en el equilibrio de poder entre las plataformas y las personas. La edad de una autorregulación inadecuada debe llegar a su fin. Los derechos de los ciudadanos deben establecerse por ley, al exigir que las empresas de tecnología se adhieran a un código de conducta redactado por el Parlamento y supervisado por un regulador independiente".

\section{CONCLUSIONES}

Resulta significativo, que muchos de los países que primero han tomado la decisión de legislar, lo han hecho por el contagio político, cultural y social que se desarrolló tras la victoria de Donald Trump en las elecciones presidenciales de EEUU.

Desde esta perspectiva, y en relación a las preguntas de investigación y a las hipótesis planteadas en este trabajo se confirma que — desde un punto de vista mediático-, los heterogéneos sistemas comparados cuentan con mecanismos reguladores específicos. Esta singularidad ha propiciado que cada país haya llegado a la situación actual de forma diferente, lo que hace más complejo poder diagnosticar y recetar tanto soluciones globales como locales ${ }^{39}$.

Del estudio de los distintos países analizados, podemos afirmar que esta coordinación — que ha de venir tanto de la UE como de sus países miembros- se ha de centrar sobre los siguientes ejes:

39 Sanmartí, J.M. et alt. (2010b). "Comparativa de modelos en las relaciones PrensaEstado: Colombia, Italia, Francia, España y Portugal”. Palabra Clave, Vol. 13, n 1. Disponible en: http://palabraclave.unisabana.edu.co/index.php/palabraclave/article/view/1638 
1. La regulación de las empresas tecnológicas como medios sociales:

- Se ha de garantizar la transparencia sobre los contenidos patrocinados, en particular la publicidad de carácter político $-\mathrm{y}$ reducir los ingresos de los vectores de desinformación-.

- Proporcionar una mayor claridad sobre el funcionamiento de los algoritmos y permitir la verificación de éstos por terceros.

- Hacer que resulte más fácil para los usuarios encontrar y acceder a fuentes distintas de noticias que representen otros puntos de vista.

- Independientemente del soporte o plataforma, la publicidad y los contenidos patrocinados deberían ser claramente distinguibles de la información y el contenido editorial.

2. La importancia de la campaña electoral como forma de expresión de la ciudadanía en democracia:

- Los artículos de publicidad política deben ser accesibles al público en un repositorio de búsqueda: quién está pagando por los anuncios, qué organizaciones están patrocinando el anuncio, quién está siendo orientado por los anuncios. Esta información es imprescindible para que la ciudadanía pueda entender el comportamiento de anunciantes individuales y se debe ejecutar independientemente de la industria de la publicidad y de los partidos políticos.

- El papel del estado como garante del proceso de elección democrática, aumentando los refuerzos de defensa, coordinación y alfabetización digital durante las campañas.

3. La defensa de la libertad de información y expresión:

- La necesidad de distinguir claramente entre iniciativas que entran dentro del código penal y aquellas que se incluyen en marcos regulatorios como el de competencia. La elección de una mirada u otra, aunque depende mucho más de la historia de las relaciones entre prensa-estado y de la forma que tienen los países de garantizar el pluralismo informativo y la libertad de expresión e información, resulta fundamental en un escenario donde lnternet se convierte en vehículo de expresión preferente de la ciudadanía.

- El etiquetado de informaciones satíricas, una clara diferenciación de los contenidos patrocinados o un reparto transparente de la publicidad institucional son mecanismos de adquisición de competencias - como consumidores de información- que nos permitirían defender con mayor conocimiento de las problemáticas que se están planteando; entre ellas la libertad de información y expresión o la circulación de contenidos. 
En cuestiones digitales se suele hablar de que es mejor no legislar que una mala legislación. Sin embargo, en este escenario de contagio legislativo no debatir sobre una posible regulación puede suponer la inclusión de leyes que poco tienen que ver con las nuevas formas de desinformación y mucho con el uso tradicional de la propaganda (interior y exterior) para restringir nuestras libertades bajo el argumento de la seguridad nacional.

$\mathrm{Al}$ mismo tiempo, es fundamental saber delegar competencias que necesitan de un marco de actuación común. Resulta interesante, al respecto, la propuesta legislativa croata que considera que el discurso del odio reúne los estándares comunes para ser regulado desde la UE.

Por último, hay que recordar que los conceptos no son estáticos sino que evolucionan, se adaptan y adquieren nuevas significaciones, pero también tienen que convivir con diferentes velocidades de adaptación. Un debate en torno a lo que se debe y no se debe legislar puede ser un buen principio para que posteriormente no tengamos que abrir otro más doloroso sobre sus disfunciones y restricciones una vez aprobada la legislación. A priori parece más razonable legislar sobre la forma en la que se presentan los contenidos que sobre los propios contenidos ${ }^{40}$.

Esta opción de apostar por una legislación en torno a la presentación de los contenidos permitiría entender la cuestión desde el punto de vista de nuestros derechos como consumidores y no sólo desde el punto de vista de nuestro derecho a la información veraz como ciudadanos.

Por último, es importante señalar que cualquier regulación debería tener en cuenta y dividir las áreas de acción y decisión antes de tomar medidas que funcionan como vasos comunicantes. Desde esta perspectiva, es posible que el informe de la Comisión de Expertos de la UE haya detenido el interés de muchos países por regular penalmente las fake news, pero a medida que más se sabe sobre el proceso resulta evidente la necesidad de adaptarse normativamente al nuevo escenario digital.

En este sentido, cualquier iniciativa necesita de distinguir entre medidas legislativas, de alfabetización digital, formativas y de acción así como entre medidas internas y externas. Sabiendo, en cualquier caso, que su actualización puede ser necesaria con cada elección.

${ }^{40}$ Magallón-Rosa, R. (2018). "Leyes fake news”. Telos. Madrid: Fundación Telefónica. Disponible en: https://telos.fundaciontelefonica.com/las-leyes-las-fake-news-problema-la-libertad-informacion-nolegislar/ 
Title:

The (no) regulation of disinformation in the European Union. A comparative perspective.

\section{Summary:}

1. INTRODUCTION. EUROPE'S DIFFERENT SPEEDS VERSUS FAKE NEWS. 2. DISINFORMATION AND FAKE NEWS. THE STATE OF THE MATTER. 3. EUROPE IN THE FACE OF DISINFORMATION. EVERY CHOICE IS A NEW CHALLENGE. 4. THE REGULATION OF DISINFORMATION IN THE DIFFERENT EU COUNTRIES. 5. HOW TO COMBAT DISINFORMATION. CONCLUSIONS AND PERSPECTIVES FOR THE FUTURE. 6. CONCLUSIONS.

\section{Resumen:}

El objetivo de este estudio es realizar un análisis comparativo en torno a las diferentes medidas tomadas por los países de la Unión Europea para combatir la desinformación. Desde esta perspectiva se analizan en primer lugar las medidas tomadas por la UE ante el nuevo escenario y, posteriormente, se estudian las medidas concretas tomadas por los diferentes países que han decidido actuar en la lucha contra las fake news y las nuevas formas de propaganda.

El estudio se centra principalmente en cuatro países: Alemania, Francia, España y Reino Unido. El trabajo pretende distinguir entre medidas legislativas, de alfabetización digital, informes, grupos de trabajo, etc. así como entre medidas internas y externas. Es decir, aquellas destinadas a monitorizar las actividades de propaganda e injerencia de terceros países en los procesos electorales.

En cualquiera de estos escenarios, la legislación digital presenta desafíos que se repiten: un modelo legislativo no adaptado a los cambios digitales, intereses de actores tradicionales que no quieren perder sus cuotas de mercado y poder, además del desconocimiento de fenómenos nuevos que rápidamente se instalan en nuestra cotidianeidad.

\section{Abstract:}

The aim of this study is to carry out a comparative analysis around the different measures undertaken by the countries of the European Union to fight against misinformation. From this perspective, we analyze the measures undertaken by the EU in the new scenario and we study the concrete actions taken by the different countries that have decided to act in the fight against fake news and disinformation.

The study focuses mainly on four countries: Germany, France, Spain and the United Kingdom. The work aims to distinguish between legislative measures, digital literacy, reports, working groups, etc. as well as between 
internal and external measures. It means, those aimed at monitoring the propaganda and interference activities of third countries in the electoral processes.

In any of these scenarios, digital legislation presents challenges that are repeated: a legislative model not adapted to digital changes, interests of traditional actors who don't want to lose their market shares and power and the unawareness of a new phenomena that quickly has been installed in our daily life.

\section{Palabras clave:}

Fake news, elecciones, regulación, desinformación, Unión Europea.

\section{Keywords:}

Fake news, elections, regulation, disinformation, European Union. 